\title{
ESTUDO DA SUPLEMENTAÇÃO COM SACAROSE PARA A PRODUÇÃO DE HIDROGÊNIO A PARTIR DE EFLUENTE DA SUINOCULTURA
}

\author{
E. C. L. SANTOS ${ }^{1}$; T. M. V. SIQUEIRA ${ }^{1}$; E. L. C. AMORIM ${ }^{1} *$ \\ 1 Universidade Federal de Alagoas (UFAL), BR 104 Km-97N, Maceió-AL, CEP 57072-970; 1Centro \\ de tecnologia-CTEC. \\ *E-mail: eduardo.lucena@uol.com.br
}

\begin{abstract}
RESUMO - Os resíduos orgânicos provenientes da atividade suinícola possuem alta concentração de matéria orgânica e podem ser utilizados como substrato para a produção de metano e hidrogênio. O objetivo do trabalho foi avaliar a produção biológica de hidrogênio a partir do efluente da suinocultura suplementado com sacarose. O reator utilizado foi anaeróbio de leito fluidificado, adotou-se um período de adaptação onde o TDH variou de 8-4h e a suplementação foi de 10 g. $\mathrm{L}^{-1}$ de sacarose. Ao passar para o TDH de $2 \mathrm{~h}$ a suplementação variou de $10-0 \mathrm{~g} . \mathrm{L}^{-1}$ de sacarose. Os resultados mostraram que o rendimento máximo de hidrogênio foi $4,90 \mathrm{molH}_{2} / \mathrm{mol}$ sacarose no $\mathrm{TDH}$ de $2 \mathrm{~h}$ com uma suplementação de 10 g.L $\mathrm{L}^{-1}$ de sacarose e a produção volumétrica máxima obtida foi de 1,47 L. $\mathrm{h}^{-1}$. $\mathrm{L}^{-1}$ no TDH de $2 \mathrm{~h}$ com 10 g. $\mathrm{L}^{-1}$ de sacarose. Os dados evidenciaram a necessidade da adição de uma fonte de carbono para uma melhor eficiência na produção biológica de hidrogênio ao utilizar efluente da suinocultura como substrato.
\end{abstract}

\section{INTRODUÇÃO}

A industrialização e o rápido crescimento demográfico vêm contribuindo com o aumento significativo dos problemas ambientais, principalmente no que se diz respeito à utilização de energia e geração de resíduo, sendo os processos industriais um dos maiores geradores de produtos e resíduos indesejáveis, os quais possuem potencial para produzir energia limpa.

Atualmente os combustíveis fosseis são as principais fontes de energia (cerca de $80 \%$ da energia mundial). A utilização desse combustível também vem ocasionando mudanças do clima global, devido à emissão de poluentes (DAS \& VEZIROGLU, 2001). Devido a isso a comunidade cientifica tem expressado grande interesse em pesquisas acerca de fontes renováveis de energia e tem dado atenção especial à produção e utilização de hidrogênio $\left(\mathrm{H}_{2}\right)$, pois esse gás pode ser produzido a partir de combustíveis fósseis, da água e de biomassa. Contudo, para que essa tecnologia seja sustentável, a sua produção deve ser realizada a partir de fontes renováveis. 


\section{9 a 22 de outubro de 2014 \\ Florianópolis/SC}

O hidrogênio é uma fonte complementar muito promissora, como fonte de energia e tem recebido mais atenção em todo o mundo nos últimos anos. O hidrogênio pode ser produzido por meio de métodosfísico-químicose biológicos. Dentre todos os métodos de produção de hidrogênio, o método biológico vem se destacando por apresentar menos gastos de energia, para que possa ser realizado, bem como o uso de temperatura ambiente, sendo economicamente mais viável especialmente se é $\mathrm{H}_{2}$ produzido por fermentação dematéria orgânica, tais como resíduos renováveis agroindustriais, que contêm carboidratos (WANG et al. 2009).

Atualmente o Brasil é considerado o $4^{\circ}$ maior produtor de carne suína, perdendo apenas para a China, União Europeia e Estados Unidos e também o $4^{\circ}$ exportador mundial, ficando atrás da União Europeia, Estados Unidos e Canadá. (ABIPECES, 2006).

O Brasil possui condições de aumentar cada vez mais sua produção de suínos, pois tem um clima favorável (tropical), mão de obra de baixo custo, facilidade para manejo e tratamento de dejetos, pelas grandes dimensões territoriais e topografia. Em se tratando da suinocultura, verifica-se que ela passou por profundas alterações tecnológicas nas últimas décadas, visando principalmente o aumento de produtividade e redução dos custos de produção. A suinocultura é uma atividade importante para a economia brasileira, pois gera emprego e renda para cerca de dois milhões de propriedades rurais.

O resíduo da suinocultura é rico em matéria orgânica, podendo ser um potencial substrato para a produção de metano e hidrogênio. Em termos energéticos, a utilização de gás hidrogênio apresenta vantagens sobre a utilização dos combustíveis fósseis convencionais, pois a combustão do hidrogênio com o oxigênio produz somente vapor de água.

O presente trabalho buscou verificar a eficiência da produção de hidrogênio a partir do efluente da suinocultura suplementado com sacarose, utilizando um reator anaeróbio de leito fluidificado, o qual foi submetido à variação de TDH e da concentração do co-substrato (sacarose).

\section{MATERIAL E MÉTODOS}

O efluente utilizado nesta pesquisa foi proveniente de uma propriedade rural localizada em Viçosa-AL. O mesmo foi coletado mensalmente e armazenado sob condições de refrigeração a temperatura de $-15^{\circ} \mathrm{C}$. O sistema avaliado foi constituído por um reator anaeróbio de leito fluidificado (RALF) em acrílico com um volume útil de $900 \mathrm{~mL}$. O efluente foi diluído à $8 \%$ (v/v) e o material suporte utilizado para garantir a aderência microbiana foi a argila expandida.

Optou-se por não ajustar o $\mathrm{pH}$ durante o experimento, com a vantagem de não se utilizar um tampão de $\mathrm{pH}$. O reator foi operado avaliando a variação do TDH de 8, 6, 4 e 2 horas, o critério utilizado para a alteração do TDH foi a estabilidade em relação a eficiência de conversão da sacarose e a formação dos produtos, como os ácidos orgânicos e o hidrogênio.O mesmo foi suplementado com uma concentração de 10 g. $\mathrm{L}^{-1}$ de sacarose, em seguida o TDH de 2 horas foi fixado, pois de acordo 
com Amorim et al. (2009) foi o TDH ótimo para produção de hidrogênio. Segundo Chen et al. (2001), o aumento da vazão pode promover condições favoráveis para a manutenção apenas de microrganismos acidogênicos e condições desfavoráveis para manutenção de arqueias metanogênicas, as quais são eliminadas do reator, visto que a velocidade de crescimento do microrganismos acidogênicos é significativametne superior a velocidade de crescimentos dos metanogênicos.

A parti daí, a suplementação passou a ser reduzida (10, 8, 6, 4, e 0 g.L ${ }^{-1}$ de sacarose). Foram realizadas algumas análises físico-químicas como DQO, pH, Alcalinidade, Ácidos Voláteis, Sólidos Totais, Sólidos Voláteis e Sólidos Fixos (APHA, 1998), Carboidratos Totais (DUBOIS et al. 1956).A produção volumétrica de hidrogênio foi medida por meio do medidor MilliGas-counter do fabricante Ritter. Ao medidor foi acoplado um recipiente contendo uma solução de hidróxido de sódio $(\mathrm{NaOH})$ com concentração de $5 \mathrm{~mol}$. $\mathrm{L}^{-1}$. Ao realizar as medições, o biogás entrou em contato com a solução de $\mathrm{NaOH}$ para que o $\mathrm{CO}_{2}$ formado ficasse retido na solução e apenas o gás hidrogênio fosse medido (AMORIM et al., 2009).

\section{RESULTADOS E DISCUSSÃO}

Na Tabela 1 podemos observar a produção volumétrica e rendimento de hidrogênio. A produção volumétrica de hidrogênio é dada em $\mathrm{L} \mathrm{deH_{2 }}$ por hora por $\mathrm{L}$ de efluente e o rendimento é expresso em mol de $\mathrm{H}_{2}$ por mol de sacarose.

Tabela 1. Produção Volumétrica (HPR), Rendimento de Hidrogênio (HY) e Eficiência de conversão em função do TDH e da Suplementação.

\begin{tabular}{ccc}
\hline Fase Experimental & $\begin{array}{c}\text { HPR } \\
\left(\mathbf{L . h}^{-\mathbf{1}} \cdot \mathbf{L}^{-\mathbf{1}}\right)\end{array}$ & $\begin{array}{c}\text { HY mol H} \\
\text { sacarose }\end{array}$ \\
\hline TDH8H/10g & $0,07 \pm 0,01$ & $2,96 \pm 2,73$ \\
TDH6H/10g & $0,37 \pm 0,13$ & $3,42 \pm 1,51$ \\
TDH4H/10g & $1,18 \pm 0,16$ & $1,58 \pm 1,07$ \\
TDH2H/10g & $1,62 \pm 0,37$ & $3,93 \pm 1,24$ \\
TDH2H/8g & $1,04 \pm 0,07$ & $1,83 \pm 0,63$ \\
TDH2H/6g & $0,93 \pm 0,00$ & $4,90 \pm 0,01$ \\
TDH2H/4g & $0,33 \pm 0,00$ & $3,36 \pm 0,13$ \\
TDH2H/2g & $0,32 \pm 0,00$ & $1,06 \pm 0,08$ \\
TDH2H/0g & $0,08 \pm 0,00$ & $0,81 \pm 0,02$ \\
\hline
\end{tabular}

Nessa pesquisa foi analisada a eficiência da produção biológica de hidrogênio, a partir da variação da suplementação com sacarose, em função do TDH. A produção volumétrica de hidrogênio aumentou com a redução do TDH. Ocorreu uma redução da produção volumétrica de hidrogênio em função da diminuição da suplementação com sacarose. Quando a suplementação com sacarose passou 
de $10 \mathrm{~g} . \mathrm{L}^{-1}$ para $0 \mathrm{~g} . \mathrm{L}^{-1}$, ambas em TDH de $2 \mathrm{~h}$, observou-se que a produção volumétrica de hidrogênio reduziu de 1,62 para $0,08 \mathrm{~L} \cdot \mathrm{h}^{-1}$. $\mathrm{L}^{-1}$. Com relação à redução do TDH de $8 \mathrm{~h}$ para $2 \mathrm{~h}$, ambos com uma suplementação de $10 \mathrm{~g}$. $\mathrm{L}^{-1}$ observou-se que a produção volumétrica sofreu um significativo aumento de 0,073 para $1,62 \mathrm{~L} \cdot \mathrm{h}^{-1} \cdot \mathrm{L}^{-1}$, conforme mostra a Tabela1.

Com relação ao rendimento de hidrogênio também sofreu variação em função da suplementação e do TDH (Tabela 1). Foi observado um acréscimo no rendimento de hidrogênio quando o TDH era de $8 \mathrm{~h} / 10 \mathrm{~g}$. $\mathrm{L}^{-1}\left(2,96 \mathrm{~mol} \mathrm{H}_{2}\right.$ mol sacarose $\left.{ }^{-1}\right)$ e passou para $6 \mathrm{~h} / 10 \mathrm{~g} . \mathrm{L}^{-1}\left(3,42 \mathrm{~mol} \mathrm{H}_{2}\right.$ mol sacarose $\left.\mathrm{s}^{-1}\right)$ e um decréscimo quando passou para o TDH de $4 \mathrm{~h} / 10 \mathrm{~g}$. L $\mathrm{L}^{-1}(1,58 \mathrm{~mol} \mathrm{H} \mathrm{mol}$ sacarose $\left.^{-1}\right)$ e um acréscimo quando passou para o TDH de $2 \mathrm{~h} / 10 \mathrm{~g}$. L ${ }^{-1}$ (3,93 mol $\mathrm{H}_{2}$. mol sacarose ${ }^{1}$ ), sendo que no TDH de $2 \mathrm{~h} / 8 \mathrm{~g}\left(1,83 \mathrm{~mol} \mathrm{H}_{2}\right.$ mol sacarose $\left.\mathrm{e}^{-1}\right)$, o rendimento de hidrogênio decresceu, mas ao passar para o $\mathrm{TDH}$ de $2 \mathrm{~h} / 6 \mathrm{~g}\left(4,90 \mathrm{~mol} \mathrm{H}_{2}\right.$. mol sacarose $\left.\mathrm{s}^{-1}\right)$ observou-se o maior rendimento de hidrogênio e nas demais fases o rendimento passou a decair TDH de $2 \mathrm{~h} / 4 \mathrm{~g}$. L $\mathrm{L}^{-1}\left(3,36 \mathrm{~mol} \mathrm{H}_{2}\right.$. mol sacarose $\left.^{-1}\right), \mathrm{TDH}$ de $2 \mathrm{~h} / 2 \mathrm{~g}$. $\mathrm{L}^{-1}\left(1,06 \mathrm{~mol} \mathrm{H}_{2}\right.$ mol sacarose $\left.{ }^{-1}\right)$ e $\mathrm{TDH}$ de $2 \mathrm{~h} / 0 \mathrm{~g} . \mathrm{L}^{-1}(3,93 \mathrm{~mol} \mathrm{H}$. mol sacarose $^{-1}$ ) respectivamente. Sendo que, na fase do TDH de $8 \mathrm{~h} / 10 \mathrm{~g}$ ao TDH de $2 \mathrm{~h} / 10 \mathrm{~g}$ foi considerada a fase de adaptação do reator, por isso, podemos observar que ocorreu uma instabilidade no rendimento de hidrogênio nesse período.

Reis \& Silva (2011) ao trabalhar com resíduo com água de esgoto sintética, contendo glicose como principal fonte de carbono observaram um aumento na produção de hidrogênio $(\mathrm{HY})$ em função da redução do TDH. Quando o TDH foi reduzido a média do HY aumentou nos dois reatores operados, variando entre 0,9 e 2,6 $\mathrm{mol} \mathrm{H}_{2} \mathrm{~mol}^{-1}$ de glicose para um TDH de $2 \mathrm{~h}$.

Barros \& Silva (2012) operando três reatores alimentados com glicose e com materiais suporte diferentes também encontrou o melhor rendimento no TDH de $2 \mathrm{~h}$ de $2,11 \mathrm{~mol} \mathrm{H}_{2} \mathrm{~mol}^{-1}$ de glicose.

Nascimbeni (2013) analisou a utilização da água residuaria sintética suplementada com sacaroseo rendimento de hidrogênio em função da concentração de suplementação com sacarose e obtido foram: rendimentos de $0,48,0,60,1,68$ e $1,49 \mathrm{~mol} \mathrm{H}_{2} \mathrm{~mol}^{-1}$ de sacarose com uma suplementação de 0,6, 1,2, 2,1 e 5,5 g/L de sacarose. Observou-se que à medida que a suplementação foi sendo retirada o rendimento de hidrogênio foi diminuindo.

Foi possível observar que o rendimento da produção de hidrogênio diminuiu conforme a diminuição da concentração de suplementação.

A Tabela 2 mostra a variação da DQO afluente, efluente e a sua conversão em todas as fases do experimento. 
Tabela 2. DQO média afluente e efluente; Eficiência de conversão.

\begin{tabular}{c|r|r|r}
\hline TDH & \multicolumn{1}{|c|}{ DQO (aflu) } & \multicolumn{1}{c}{ DQO (eflu) } & \multicolumn{1}{|c}{$\begin{array}{c}\text { Eficiência } \\
(\boldsymbol{\%})\end{array}$} \\
\hline TDH8h/10g & 13504,50 & 7347,46 & 45,59 \\
$\mathbf{T D H 6 h} / \mathbf{1 0 g}$ & 13504,50 & 11031,78 & 18,31 \\
$\mathbf{T D H 4 h} / \mathbf{1 0 g}$ & 13504,50 & 11607,84 & 14,04 \\
$\mathbf{T D H 2 h} / \mathbf{1 0 g}$ & 13504,50 & 13273,24 & 1,71 \\
$\mathbf{T D H 2 h} / \mathbf{8 g}$ & 12105,03 & 11522,29 & 4,81 \\
$\mathbf{T D H 2 h} / \mathbf{6 g}$ & 8537,57 & 8318,99 & 2,56 \\
$\mathbf{T D H 2 h} / \mathbf{4 g}$ & 6764,50 & 6596,99 & 2,48 \\
$\mathbf{T D H 2 h} / \mathbf{2 g}$ & 3590,65 & 3036,66 & 15,43 \\
$\mathbf{T D H 2 h} / \mathbf{0 g}$ & 1817,57 & 1365,15 & 24,89 \\
\hline
\end{tabular}

A DQO afluente variou entre $13.504,50 \mathrm{e} 1.817,98 \mathrm{mg} \cdot \mathrm{L}^{-1}$ e a efluente variou entre 13.273,24 e 1365,15 mg. $\mathrm{L}^{-1}$ com uma eficiência de conversão variando entre 56,84 a 9,87\% .

A eficiência máxima na conversão de matéria orgânica foi de $45,59 \%$ no TDH de $8 \mathrm{~h} / 10 \mathrm{~g}$ e a menor foi de $1,71 \%$ no $\mathrm{TDH} 2 \mathrm{~h} / 10 \mathrm{~g}$.

\section{CONCLUSÕES}

Este estudo demonstrou a viabilidade de melhorar a produção de hidrogênio a partir de produção biológica por fermentação de efluente de suinocultura suplementado com sacarose. O presente estudo mostrou a aplicabilidade do RALF para a produção de hidrogênio.

Os resultados mostraram que a diminuição da suplementação tem o feito de diminuir a taxa de produção volumétrica (HPR) e o rendimento (HY). O reator obteve uma produção volumétrica máxima de 1,62 L. $\mathrm{h}^{-1}$. $\mathrm{L}^{-1}$ e um rendimento de 4,90 mol $\mathrm{H}_{2}$ mol sacarose $\mathrm{m}^{-1}$. Também foi observado que os valores de HY e HPR aumentaram com a diminuição do TDH de $8 \mathrm{~h}$ para $2 \mathrm{~h}$.

Os resultados do estudo indicaram que, a sacarose como suplemento pode proporcionar não só nutrientes, mas tem a capacidade de manter o $\mathrm{pH}$ ideal para a produção de hidrogênio, enquanto que serve como co-substrato para aumentar o rendimento de hidrogênio.

\section{REFERÊNCIAS}


ABIPECS - ASSOCIAÇÃO BRASILEIRA DA INDÚSTRIA PRODUTORA E EXPORTADORA DE CARNE SUÍNA. Site corporativo. Disponível em http://www.abipecs.org.br. Acessado em 17 de outubro de 2013.

AMERICAN PUBLIC HEALTH ASSOCIATION (APHA); AMERICAN WATER WORKS ASSOCIATION (AWWA); WATER ENVIRONMENT FEDERATION (WEF). Standard methods for the examination of water and wastewater. 19th ed. Washington 1998.

AMORIM et al. Anaerobic Fluidized Bed Reactor with Expanded Clay as Support for Hydrogen Production thorough Dark Fermentation of Glucose. International Journal of Hydrogen Energy, v. 34, p. 783-790, 2009.

BARROS, A. R; SILVA, E. L.Hydrogen and ethanol production in anaerobic fluidized bed reactors: Performance evaluation for three support materials under different operating conditions.Biochem. Engin. J.v. 61, p. 59-65, 2012.

CHEN, C. C.; LIN, C. Y.; CHANG, J. S. Kinetics of hydrogen production with continuos anaerobic cultures utilizing sucrose as the limiting substrate. Ap. Micro. Biotech.,v.57, p. 56-64,2001.

DAS, D.; VEZIROGLU T.N. Hydrogen production by biological processes: a survey of literature. International J. of Hyd. Ener., v .26, p. 13-28, 2001.

DUBOIS, S.M. et al. Colorimetric Methods for determination of sugar and related substance. Analy. Chem., v. 228, p. 13-21. 1956.

NASCIMBENI, F. A. Avaliação da produção biológica de hidrogênio em reator em batelada sob condições termófila. 2013. 74 f. Monografia (Graduação em Engenharia Ambiental) Universidade de São Paulo, São Carlos, 2013.

WANG, J.L; Wan W. Factors influencing fermentative hydrogen production: a review. Int J. Hyd. Ener.; v. 34, n. 2, p. :799-811, 2009. 